Please send trade news information and illustrations to Arveen Bajaj at the BDJ, 64 Wimpole Street, London W1G 8YS.

Trade news is supplied as a service to the reader and does not imply endorsement by the BDJ. Normal and prudent research should be exercised before purchase of use of any product mentioned.

\section{The soft touch}

Ansell has created a new examination glove designed specifically for dentists and which claims to address the problems associated with daily use.

MICRO-TOUCH HydraCare gloves provide protection against micro-organisms and combine a low allergenic profile with the cooling properties of chamomile. Added to this is a moisturizing agent. They feature a textured surface, enabling a more secure grip of instruments, and have a unique design for a perfect fit for all hand shapes and sizes.

Reader response number 50

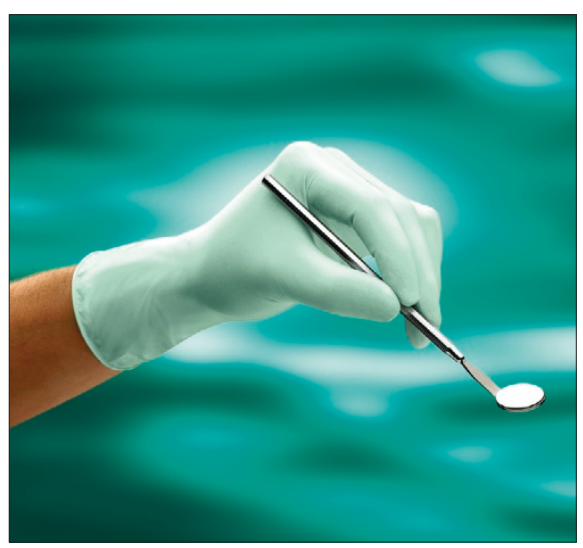

Rubber dam range

Henry Schein offer a range of rubber dams of all types, including thin gauge which is easier to place when gingival retraction is not critical, medium gauge, a general purpose material that can be used in most procedures, or heavy gauge which provides stronger resistance to tearing and greater gingival retraction.

Reader response number 51

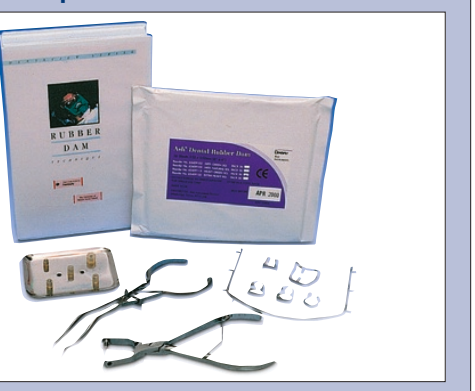

\section{TRADE NEWS}

\section{Starting young}

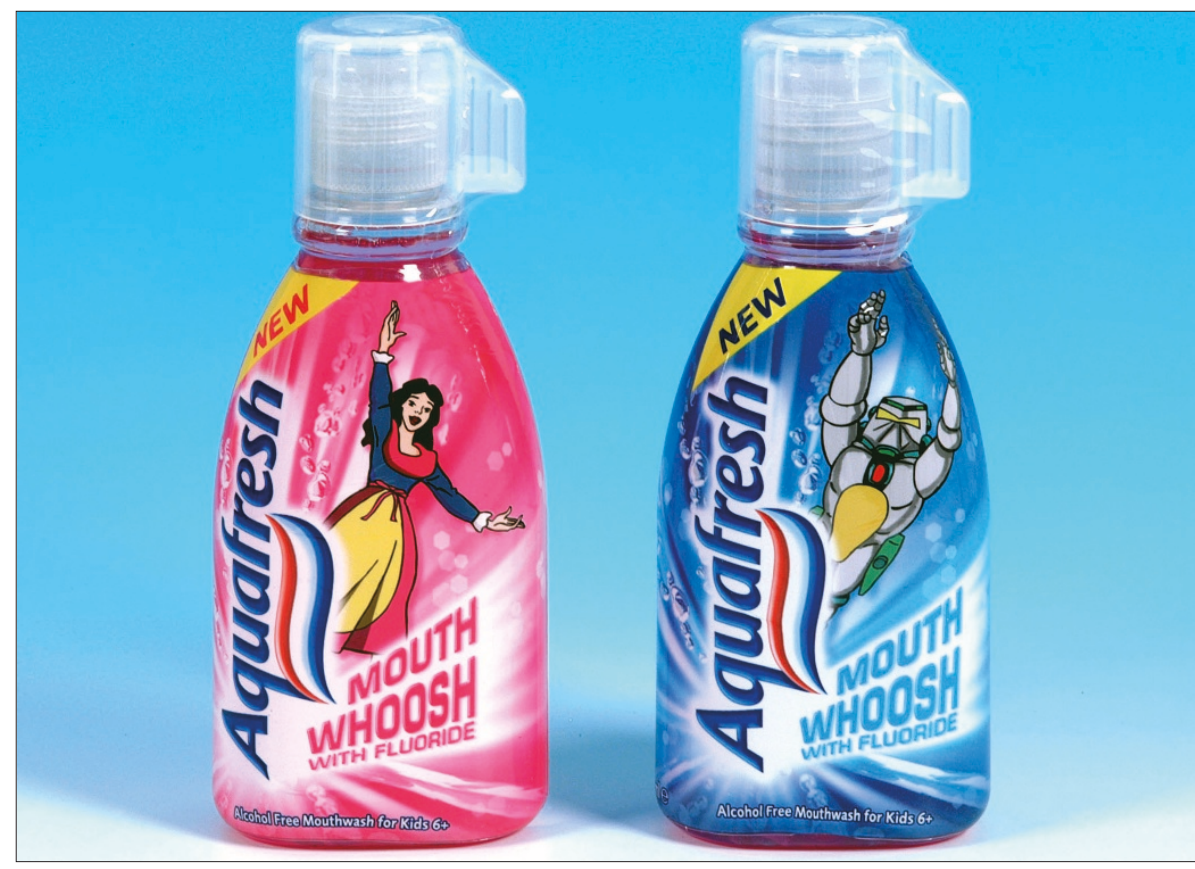

Aquafresh has launched a new mouthwash aimed at the younger user. Aquafresh Mouth Whoosh is an antibacterial, alcohol-free, fluoride mouthwash designed to appeal to boys and girls aged 6 to 12. The product features Clipon Friends characters Princess Isabella and Spaceman Spectrum, in their own different coloured easily identifiable packaging. Reader response number 52

\section{New separator launched}

The new Metasys Compact Dynamic Amalgam Separator distributed by PuriDent UK facilitates separation at source of amalgam waste from both the suction hoses and spittoon, and complies with both EN ISO and DIN specifications.

A modern single unit amalgam separator, it divides air, liquid and amalgam and because of its small size and modular design, it can be fitted into most brands of dental cuspidor.

It features simple technology, a large collecting vessel and built-in warning systems. The latter advise the practice when it is time to replace the vessel, which is simple to change, disinfect and seal before being returned for processing.

It requires no maintenance except disinfecting.

Reader response number 53

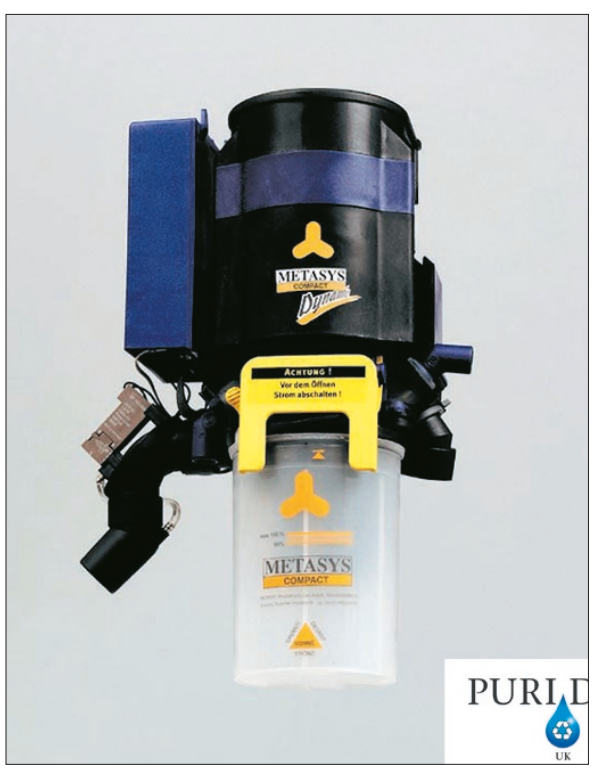

